\title{
Predictores cognitivos de la intención de práctica y la percepción de las relaciones en el deporte: análisis de la pasión como mediadora \\ Cognitive predictors of the intention of practice and the perception of relationships in sports: analysis of passion as a mediator \\ David Pérez García, José Francisco Guzmán Luján \\ Universidad de Valencia (España)
}

\begin{abstract}
Resumen. Este estudio analizó el papel de la inteligencia en el juego y la motivación como predictores de la intención de práctica deportiva y las relaciones sociales. Asimismo examina el papel de la motivación autodeterminada y la pasión (armoniosa y obsesiva) como mediadoras de esta relación, incorporando la percepción de conflicto. Participaron 180 jugadores adolescentes de fútbol, baloncesto y balonmano, de ambos géneros (124 chicos y 56 chicas) y de edades comprendidas entre 15 y 19 años (M=15,9; S=1,029). Se llevó a cabo un análisis correlacional y análisis SEM (Structural Equation Modeling) de dos modelos estructurales, uno con la pasión armoniosa y otro con la pasión obsesiva. El ajuste del modelo de la pasión armoniosa fue mejor que el de la pasión obsesiva. En el primero la inteligencia en el juego y la motivación autodeterminada predijeron la pasión armoniosa, y ésta predijo positivamente las relaciones con la familia y el entrenador, y la intención de práctica deportiva. En el segundo sólo la inteligencia en el juego predijo la pasión obsesiva que a su vez predijo positivamente sólo la intención de práctica. Los resultados apoyan el uso de estrategias de intervención para mejorar la inteligencia en el juego y desarrollar la motivación autodeterminada y la pasión armoniosa, dada su capacidad para predecir la intención de práctica y las relaciones de los deportistas.
\end{abstract}

Palabras clave: Inteligencia, motivación, deportistas, conflicto, análisis estructural.

\begin{abstract}
This research analyzed the role of intelligence in the game and motivation as predictors of the intention of practicing sports and social relations. It also examines the role of self-determined motivation and passion (harmonious and obsessive) as mediators of this relationship, incorporating the perception of conflict. 180 teenage soccer, basketball, and handball players of both genders (124 boys and 56 girls) aged between 15 and 19 years old participated in the study. Correlation analysis and SEM (Structural Equation Modelling) analysis of two structural models were conducted, one with harmonious passion and the other with obsessive passion. The fit of the harmonious passion model was better than the obsessive passion model. In model 1 the intelligence in the game and self-determined motivation predicted harmonious passion positively, which was also positively related with family and coach relationship, and sport practice intention. In model 2 only intelligence in the game predicted the obsessive passion which positively predicted intention to practice sport. These results support the use of intervention strategies to improve intelligence in the game and develop self-determined motivation, as well as it highlights the importance of harmonious passion for predicting exercise behavior and athletes' relationships.
\end{abstract}

Keywords: Intelligence, motivation, sportsmen, conflict, structural analysis.

\section{Introducción}

El entrenamiento en el ámbito deportivo va orientado a conseguir un mayor rendimiento en el deportista, de forma que una alta cantidad y calidad de práctica deliberada, puede explicar la excelencia deportiva (Ericsson, 2008). Varios estudios lo avalan en diferentes deportes (Baker, Côté \& Abernethy, 2003; Ward, Hodges, Williams \& Starkes, 2004), estableciéndose así una relación entre el número de horas practicadas y el nivel de pericia, de forma que los deportistas que más entrenan son aquellos que mayor rendimiento alcanzan. Del mismo modo, los deportistas creen que el éxito se logra mediante el esfuerzo realizado en los entrenamientos y las competiciones (Abraldes, Granero-Gallegos, BaenaExtremera, Gómez-López, \& Rodríguez-Suárez, 2016). ¿Pero qué factores pueden influir sobre la persistencia en la práctica deportiva y cómo se relacionan entre ellos?

La capacidad para actuar de manera inteligente y tomar las decisiones apropiadas está siempre sometida a los vaivenes de la disposición psicológica de los jugadores, a su resistencia mental para afrontar los retos (Hatfield \& Kerick, 2007; Jones, 2012), esto es algo que se aprende, y que los

Fecha recepción: 02-05-18. Fecha de aceptación: 12-01-19

David Pérez García

david_nizer_6@hotmail.com jugadores de mayor nivel y mayor experiencia, muestran de forma más clara que los jugadores de niveles inferiores (Baker, Coteì \& Abernethy, 2003), ya que, en general, llevan realizando la práctica deportiva durante más tiempo. Es por ello, que se puede afirmar que no se concibe un alto rendimiento deportivo de un jugador en un deporte sin una vinculación a largo plazo. Tener éxito en el deporte reclama conocer e interactuar de forma efectiva e inteligente en el contexto en el que se tiene que desenvolver (Brown, Gould \& Foster, 2005). Sternberg (1985) define la inteligencia como una actividad mental dirigida hacia la adaptación intencional, selección o transformación de entornos del mundo real relevantes en la propia vida. El mismo autor, destaca la existencia de una inteligencia práctica o contextual como parte de su modelo triárquico, resaltando que dicha inteligencia debería ser evaluada de forma contextual. Los teóricos de la inteligencia contextual (Cianciolo, Matthew, Sternberg \& Wargner, 2006; Sternberg, 2000) defienden que es aquella que se pone en acción cuando la persona tiene que adaptarse, seleccionar y acomodarse al entorno para obtener objetivos personalmente valiosos o el éxito en un ámbito de trabajo, es decir, cuando tiene que actuar de manera competente. Lo que reclama el deporte es una inteligencia dispuesta a solucionar problemas prácticos y concretos que implican movimientos precisos y controlados en un contexto muy específico (Gardner, 1983; Terenzini, 1993). Con respecto a lo indicado anteriormente, Ruiz, Graupera \& García (2014) desarrollaron la Inteli- 
gencia Contextual Percibida en el Deporte, variable que va a ser utilizada en esta investigación (en este estudio la inteligencia en el juego y la inteligencia contextual percibida en el deporte significan lo mismo), definiéndola como la capacidad para conocer la dinámica de la actividad, comprender las competencias que dichos deportes reclaman en cada momento, saber cómo lograr el éxito y conocer qué aspectos son críticos para alcanzar ese objetivo. Para ello es de vital importancia manejar recursos cognitivos para adaptarse a cada situación (elementos de autocontrol y autorregulación) lo que generaría confianza en las capacidades propias. Los deportistas más inteligentes en el juego tienen más probabilidades de tener éxito deportivo (Sternberg et al., 2000). Varios estudios la mencionan como Inteligencia Práctica o Contextual en el Deporte (Sternberg et al., 2000; Ruiz et al., 2014; Pérez, Navia, Espín, Coll \& Nieto, 2015). Dicha inteligencia reclama el empleo coordinado y preciso de movimientos corporales, estando estrechamente relacionada con la noción de inteligencia Cinestésico Corporal de Gardner (1983). En este sentido, las personas que se perciben más inteligentes en el juego o más competentes en su campo de actuación, en este caso el deporte, están más intrínsecamente motivados a persistir y rendir en el mismo (Deci \& Ryan, 1985; Bandura, 1997). Ser competente y percibirse competente son las dos dimensiones que interactuìan en el rendimiento deportivo que la praìctica y el entrenamiento tienen la obligacioìn de promover y mantener (Ommundsen \& Vaglum, 1997). Los deportistas disfrutan más porque tienen una mejor comprensión de la actividad, es por ello que van a desarrollar motivaciones más autodeterminadas.

La motivación es una variable importante para el rendimiento y la práctica deportiva, siendo relevante para la consecución de las metas y de los objetivos que se proponen los deportistas (Medrano \& Mateos, 2018). Desde la Teoría de laAutodeterminación (TAD) (Deci \& Ryan, 2000; Vallerand $\&$ Houlfort, 2003), se propone que en contextos que satisfagan las necesidades básicas de competencia, autonomía y relaciones sociales, las personas van a realizar su actividad por razones más autónomas (mayor motivación autodeterminada), debido a la satisfacción innata que produce su práctica, lo cual lleva a desarrollar una pasión armoniosa por la actividad. Varias investigaciones muestran que las principales razones en la práctica del deporte son intrínsecas y las menos importantes son extrínsecas. (Ruiz-Juan \& Zarauz, 2012; Zubiaur \& Riego, 2015; Gutiérrez, Tomas, \& Calatayud, 2018; Muñoz et al., 2018). Por otro lado, cuando el contexto ejerce mayor control sobre el deportista, se fomenta una motivación menos autodeterminada, caracterizada por motivaciones más externas, que lleva a desarrollar una pasión más obsesiva.

La motivación intrínseca, por otro lado, también se relaciona con una mayor intención de persistencia en la práctica deportiva (Franco, Coterón, Gómez, Brito \& Martínez, 2017) y con una menor percepción de conflicto entre la práctica deportiva, el contexto escolar y la amistad (Medrano \& Mateos, 2018). Lo que se traduce, en menor abandono de la actividad deportiva (Almagro \& Paramio-Pérez, 2017; Almagro, Sáenz-López \& Moreno, 2010; Guzmán \& Kingston, 2012; Luckwü \& Guzmán, 2011; Pelletier, Fortier, Vallerand, \& Brière, 2001). A su vez, el abandono ha sido predicho por la motivación externa, la amotivación y la percepción de conflicto con otras actividades (García, Leo, Martín \& Sánchez, 2008; Luckwu \& Guzmán, 2011; Pelletier et al., 2001; Rodríguez-Allen, 2000).

La pasión (armoniosa y obsesiva), razón de los dos modelos estructurales planteados en este estudio, tiene que ver con la forma en que se practica una actividad (el objeto de la pasión), y la forma en que ésta es vivida por la persona, formando parte de su identidad. Se conceptualiza como una fuerte preferencia de la persona hacia una actividad que considera importante en su vida, que la autodefine, le gusta y a la cual dedica tiempo y energía (Vallerand, 2008, 2010). Siguiendo a este autor, la pasión armoniosa conduce a un mayor afecto positivo y menor afecto negativo que la pasión obsesiva durante el tiempo de dedicación a la tarea, debido a que la internalización de la actividad contribuye a que la persona participe en ésta de una manera más flexible y viva la experiencia más plenamente. Los dos tipos de pasión pueden llevar a tener niveles elevados de actividad física en los deportistas, pero aquellos en los que predomina la pasión armoniosa, tendrán una mejor calidad en las relaciones interpersonales, puesto que lleva a las personas a invertir tiempo y energía para mantenerlas y mejorarlas (Vallerand, 2008). La pasión armoniosa está negativamente relacionada con los conflictos interpersonales. Tener una pasión armoniosa hacia una actividad podrá promover la calidad de la relación e incluso proteger contra desacuerdos y malentendidos con las demás personas de la actividad. Las personas con una pasión armoniosa hacia una actividad pueden tener la capacidad de acoplarse con otras personas de una forma más eficaz porque se dedican a realizar la actividad apasionada de una forma más abierta y flexible. (Lafreniére, Jowett, Vallerand, Donahue \& Lorimer, 2008; Philippe, Vallerand, Houlfort, Lavigne \& Donahue, 2010; Séguin-Le’vesque Laliberte, Pelletier, Vallerand \& Blanchard, 2003; Vallerand et al., 2008). Por otro lado, la pasión obsesiva implica costes personales como deterioro de las relaciones interpersonales, conflictos con otras actividades y compromisos fallidos de trabajo (Philippe, Vallerand, Houlfort, Lavigne, \& Donahue, 2010). Las relaciones interpersonales que crea el deportista con la familia, los amigos y el entrenador son clave para experimentar sensaciones positivas durante y después de la práctica deportiva (Vallerand, 2012), lo que le podría llevar a una alta participación y persistencia a largo plazo (Vallerand, 2010). Por lo que, esas relaciones se consideran clave para seguir realizando ejercicio físico (Bermejo, Almagro \& Rebollo, 2018). Tanto la familia, los amigos como el entrenador pueden ser considerados como los principales agentes de socialización deportiva (Escartí, 2003). Estas relaciones son fundamentales para el desarrollo de una adecuada práctica y un buen rendimiento deportivo (Olympiou, Jowett \& Duda, 2008). Pudiendo limitar que haya una vinculación a largo plazo si no se establecen buenas relaciones con las personas que rodean ese contexto. Tanto las relaciones sociales como la intención de práctica deportiva, ocupan un papel fundamental en el compromiso con la actividad.

Específicamente no se han encontrado estudios que analicen la percepción de inteligencia en el juego en el deporte con estas variables psicológicas descritas. Relacionando la 
inteligencia en el juego con variables motivacionales (motivación autodeterminada, pasión, percepción de conflicto), el bienestar social (relaciones sociales) y la adherencia al deporte (intención de práctica deportiva). Es por ello que consideremos de vital importancia la realización de esta investigación, para poder dar respuesta a esta relación. Existen estudios que han relacionado varias variables con diferentes tipos de inteligencia (Gardner, 1983, 2008, 2010). Dentro del concepto de inteligencia, muchas de las investigaciones de los últimos años van ligadas a la inteligencia emocional, junto a diferentes variables motivacionales (Alonso, Rodríguez, Pérez \& González, 2016; Fernández-Ozcorta,Almagro \& López, 2017; Martín, Guzmán \& De Benito, 2018). Este estudio surge como necesidad para seguir indagando sobre la inteligencia en el juego, como ya han realizado otros estudios (Ruiz, Navia, Miñano, García \& Palomo, 2015; Ruiz, García, Palomo, Navia \& Miñano, 2014; García, 2009; De la Vega, Del Valle, Maldonado \& Moreno, 2008; Elferink-Gemser, Visscher, Richard \& Lemmink, 2005).

Los objetivos del presente estudio fueron, analizar el papel mediador que tiene la pasión sobre la inteligencia en el juego, la motivación autodeterminada y las relaciones para predecir la intención de práctica. Se realizaron dos modelos estructurales, uno con la pasión armoniosa y otro con la pasión obsesiva (Figuras 1 y 2), en el que se integran las relaciones entre las diferentes variables. Averiguar qué modelo presenta mejor ajuste, para identificar la pasión con mejores implicaciones para la práctica deportiva y analizar el papel que tiene la inteligencia en el juego sobre la intención de práctica, la motivación y el bienestar social y deportivo del jugador.

Se hipotetizó que la pasión armoniosa actuaría como mediadora entre la motivación autodeterminada y la intención de práctica. También, entre la inteligencia en el juego y la intención de práctica. La pasión obsesiva en cambio mediaría entre la inteligencia en el juego y la intención de práctica pero no entre la motivación y la intención de práctica. A su vez, el modelo de pasión armoniosa se relacionará mejor con el resto de las variables y tendrá un mejor ajuste que el modelo de pasión obsesiva.

Por último, se hipotetizó que la inteligencia en el juego predeciría positivamente la pasión (armoniosa y obsesiva) y la motivación autodeterminada. La motivación autodeterminada predeciría positivamente sólo la pasión armoniosa, mientras que no predeciría la pasión obsesiva o lo haría negativamente, también predeciría positivamente la intención de práctica y negativamente la percepción de conflicto. La pasión armoniosa predeciría positivamente las relaciones interpersonales y la intención de práctica y negativamente la percepción de conflicto, mientras que la pasión obsesiva no predeciría las relaciones interpersonales o lo haría negativamente, sí predeciría la intención de práctica y predeciría positivamente la percepción de conflicto. Por último, la percepción de conflicto predeciría negativamente la intención de práctica. Se planteó que la variable inteligencia, influye sobre el resto de variables cognitivas, incluyendo en un mismo modelo, la inteligencia en el juego, las variables motivacionales y el bienestar del deportista para que la experiencia deportiva del deportista sea positiva y a largo plazo, teniendo así un alto compromiso con el deporte.

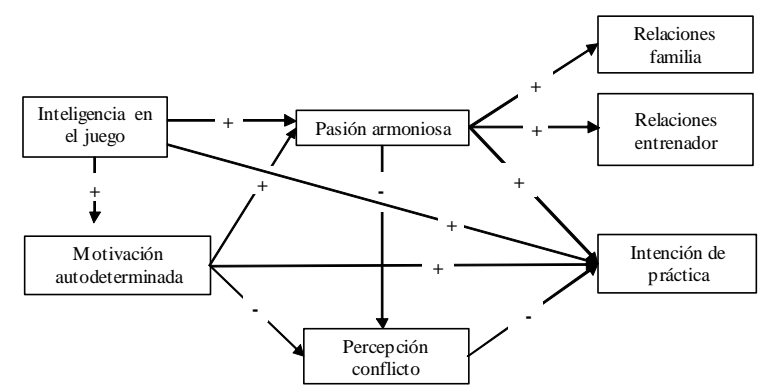

Figura 1. Modelo de relaciones estructurales propuesto para la pasión armoniosa.

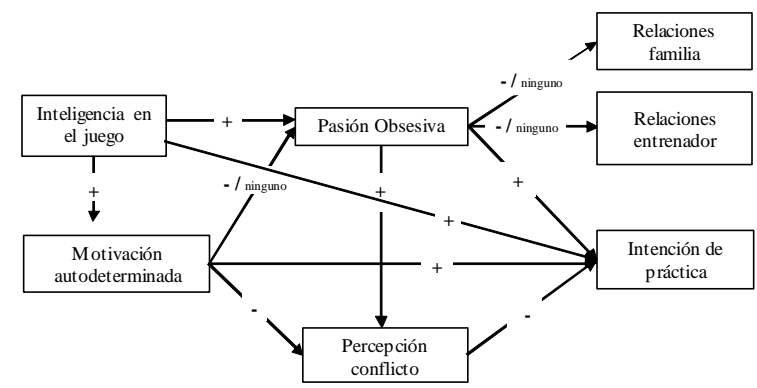

Figura 2. Modelo de relaciones estructurales propuesto para la pasión obsesiva.

\section{Métodología}

\section{Diseño}

El estudio presenta un diseño expost-facto-retrospectivo de grupo único (Fontes et al., 2010), con una finalidad explicativa, puesto que analiza la relación funcional entre las variables.

\section{Participantes}

Participaron en el estudio 179 deportistas españoles de ambos sexos. El 69\% fueron chicos y el 31\% chicas, con tiempo de práctica de más de un año y edades comprendidas entre 15 y 19 años ( $M=15,91 ; S=1,029)$. Los participantes del estudio pertenecían a las categorías cadete y juvenil de las modalidades deportivas de fútbol (40\%), baloncesto (20\%) y balonmano (40\%), de la provincia de valenciana.

\section{Instrumentos y propiedades psicométricas}

Se comprobó la normalidad de los mismos (KolmogorovSmirnov) y la consistencia interna de las diferentes escalas ( $\alpha$ de Cronbach). La motivación hacia el deporte se midió a través de la la Revised Sport Motivation Scale, elaborada por Pelletier, Rocchi, Vallerand, Deci \& Ryan (2013). Traducida y validada al castellano de Guzmán, Carratalá, García-Ferriol \& Carratalá (2006). La escala estaba formada por 18 ítems que medían: Motivación Intrínseca, Motivación Extrínseca Integrada, Motivación Extrínseca Identificada, Motivación Extrínseca Introyectada, Motivación Extrínseca de Regulación Externa, y Amotivación, estando cada una de ellas medida por tres ítems. Las respuestas a los diferentes ítems estaban representadas en una escala tipo Likert de siete puntos, donde (1) representaba «No corresponde en absoluto» y (7) «Corresponde completamente». La escala está compuesta por ítems como: «Practico mi Deporte... Porque me gusta aprender más acerca de mi Deporte». La fiabilidad de las dimensiones mostró valores alfa superiores a $(\alpha=.70)$ en todas las dimensiones excepto en la Motivación Extrínseca Introyectada (MEI) $(\alpha=$.60). La fiabilidad de la MEI no fue satisfactoria, sin embargo, valores alfa ligeramente inferiores 
a .70 pueden aceptarse cuando el número de ítems de la dimensión es bajo (en este caso sólo 3 ítems) o es bajo el número de participantes (Cortina, 1993). Para un mejor análisis, calculamos el Índice de Autodeterminación de la motivación tal y como es propuesto por Vallerand (2007). Asimismo, la MEI tiene bajo peso en comparación con otras dimensiones de la motivación en la ecuación utilizada para calcular el Índice de Autodeterminación de la motivación (Vallerand, 2007): ((MI x 3) + (ME Integrada x 2) + ME Identificada - ME Introyectada-(ME de Regulación Externa x 2)-(Amotivación x 3). Por ello, consideramos apropiado no excluir la MEI del cálculo de este índice. Según este autor, un valor positivo obtenido en este índice representa un perfil motivacional relativamente autodeterminado en la muestra, en cambio un resultado negativo puede representar un perfil motivacional con bajos niveles de autodeterminación.

Para medir la pasión en el deporte, se utilizó la adaptación española de la Escala de Pasión al Ámbito Deportivo (Pedrosa, García-Cueto, Torrado \& Arce, 2017). Consta de 14 ítems distribuidos en dos subescalas: Pasión armoniosa y pasión obsesiva. Las respuestas se dieron en una escala tipo Likert de 1 a 7 . La fiabilidad interna de los factores presentó valores aceptables tanto para la pasión obsesiva $(\alpha=.84)$ como para la pasión armoniosa $(\alpha=.84)$.

La Inteligencia en el juego se midió a través del Cuestionario de Inteligencia Contextual Percibida en el deporte(ICD). Este cuestionario fue desarrollado y validado por (Ruiz et al., 2014). Consta de 24 ítems y tres subescalas: (1) Inteligencia Anticipatoria (IA; e.g., «Soy capaz de anticipar las acciones de mis oponentes»), que destaca por la presencia de la intuición, captación de señales o la vigilancia previa a la actuación; (2) Inteligencia Táctica (IT; e.g., «Suelo elegir la técnica apropiada a cada situación de la competición»), referida a la competencia para dar las soluciones oportunas a las situaciones con las que se enfrenta el deportista; (3) Inteligencia Competitiva (IC; e.g., «Reacciono rápidamente a los cambios en la competición»), que refleja la capacidad de poner en acción los planes establecidos en la competición. Su valoración se presenta en una escala tipo Likert de diez puntos, desde el 1 «Totalmente en desacuerdo» a 10 «Totalmente de acuerdo». Con ello se puede obtener una medida global de (ICD). Debido a los intereses del estudio, se utilizó una dimensión de primer orden, la cual denominamos «Inteligencia en el juego» que mostró una fiabilidad adecuada ( $\alpha=.85)$.

Para medir la intención de práctica deportiva se utilizó la escala de intención de práctica deportiva y percepción de conflicto de Guzmán \& Kingston (2011), compuesta por ocho ítems (cuatro ítems para cada dimensión). Mostró una fiabilidad adecuada tanto para la intención de práctica deportiva $(\alpha=$.74) como para la percepción de conflicto $(\alpha=.80)$. Se contestó en una escala tipo Likert de siete puntos.

Para valorar las relaciones interpersonales se utilizó una escala compuesta por 9 ítems. Tres ítems para cada uno de los siguientes entes sociales: la familia y el entrenador. Con el cual se obtuvo una valoración general de la calidad de la relación personal de cada ente social. (Ítem 1: «Tengo buena relación con mi entrenador/ familia»; Ítem 2: «Me siento comprendido por mi entrenador/familia»; Ítem 3: «Suelo tener disputas con mi entrenador/familia por el deporte»). Su valoración se presenta en una escala tipo Likert de cinco puntos, desde el 1 «No corresponde en absoluto» a 5 «corresponde totalmente». Mostró una fiabilidad de $\alpha=.74$ para la familia y de $\alpha=.81$ para el entrenador. El análisis factorial confirmatorio mostró que los índices de ajuste fueron aceptables ( $\chi 2$ / $\mathrm{gl}=2.10 ; \mathrm{IFI}=.93 ; \mathrm{TLI}=.95 ; \mathrm{CFI}=.93 ; \mathrm{NFI}=.92 ; \mathrm{RMSEA}=$ $.06)$.

\begin{tabular}{|c|c|c|c|c|c|c|c|}
\hline Dimensiones & Alfa & Media & $\begin{array}{l}\text { Desviación } \\
\text { Típica }\end{array}$ & Asimetría & Curstosis $^{K}$ & $\begin{array}{c}\text { Kolmogorov } \\
\text {-Smirnov }\end{array}$ & $\begin{array}{l}\text { Número } \\
\text { Ítems }\end{array}$ \\
\hline M. Intrínseca & .79 & 5.78 & 1.14 & -1.15 & 1.01 & $<.001$ & 3 \\
\hline M. Integrada & .76 & 5.52 & 1.30 & -1.06 & 1.05 & $<.001$ & 3 \\
\hline M. Identificada & .77 & 5.53 & 1.29 & -.96 & .54 & $<.001$ & 3 \\
\hline M. Introyectada & .60 & 3.71 & 1.70 & .18 & -.73 & .004 & 3 \\
\hline M.R. Externa & .75 & 2.32 & 1.43 & 1.03 & .23 & $<.001$ & 3 \\
\hline Amotivación & .72 & 2.25 & 1.37 & 1.37 & 1.56 & $<.001$ & 3 \\
\hline P. Armoniosa & .84 & 5.55 & 1.01 & -.94 & .75 & $<.001$ & 7 \\
\hline P. Obsesiva & .84 & 4.27 & 1.28 & -.19 & -.43 & .010 & 7 \\
\hline Inteligencia en el juego & .85 & 7.02 & 1.30 & -.111 & .032 & .017 & 24 \\
\hline I. Práctica & .74 & 6 & 1.08 & -1.55 & 2.61 & $<.001$ & 4 \\
\hline P. Conflicto & .80 & 2.65 & 1.31 & .54 & -.69 & $<.001$ & 4 \\
\hline Relación Familia & .74 & 4.44 & 1.02 & -1.86 & 2.67 & $<.001$ & 3 \\
\hline Relación Entrenador & .81 & 4.17 & .95 & -.61 & 1.54 & $<.001$ & 3 \\
\hline
\end{tabular}

\section{Procedimiento}

Las variables que consideramos en nuestro estudio fueron: la inteligencia contextual en el juego, la motivación autodeterminada, la pasión en el deporte, la percepción de conflicto entre el deporte y las demás actividades, las relaciones con la familia y el entrenador, y finalmente la intención de práctica deportiva. Todos ellas se midieron mediante el uso del cuestionario. Se contactó previamente con los entrenadores para verificar su disponibilidad para participar en la investigación. Tras una entrevista con los entrenadores éstos se encargaron de administrar los cuestionarios a sus deportistas. Éstos últimos accedieron a participar libremente en el estudio sabiendo que los datos personales no serían revelados y los resultados obtenidos no serían utilizados para otra finalidad distinta a la investigación. Se cumplieron los criterios éticos para este rango de edad y firmaron un consentimiento informado con dicha información, de acuerdo con la declaración de Helsinki de La Asociación Médica Mundial (AMM) del 2004. Todos los participantes dispusieron del tiempo necesario para contestar a todos los ítems de las escalas. El promedio de tiempo utilizado fue de 20 minutos aproximadamente.

\section{Análisis de los datos}

Para el procesamiento de los datos se usaron los programas estadísticos IBM SPSS Statistics, versión 20 y AMOS versión 22. Las variables no mostraron ajuste a la normalidad por lo que se aplicaron procedimientos de normalización a través de logaritmos y se calcularon las puntuaciones $\mathrm{Z}$, con la que se realizaron los cálculos estadísticos. Seguidamente se estudiaron las correlaciones bivariadas entre las variables del estudio. Posteriormente se analizaron mediante el método de máxima verosimilitud y con 1000 muestras de bootstrapping los dos modelos estructurales hipotetizados, uno incluyendo la pasión armoniosa y en el otro la pasión obsesiva. Para cada modelo se indicó la normalidad multivariada, los coeficientes â y sus probabilidades, las varianzas explicadas y los índices de ajuste del modelo. Finalmente se realizó un análisis de mediación de cada tipo de pasión mediante el test de Sobel con 1000 muestras de bootstrapping utilizando la macro/interfaz Process para SPSS como indican Muñoz \& González (2017). 


\section{Resultados}

\section{Prueba de normalidad y Análisis correlacional}

La prueba de Kolmogorov-Smirnov para las distintas variables indicó que todas se distribuían de forma no paramétrica $(p<.05)$, por lo que se aplicaron procedimientos de normalización a través de logaritmos y se calcularon las puntuaciones Z. Para el análisis correlacional utilizamos la prueba R Pearson.

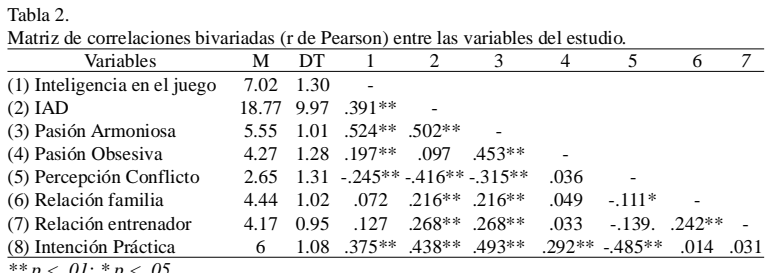

La inteligencia en el juego se relacionó negativamente con la percepción de conflicto $\left(\mathrm{r}=-.245^{* *}\right)$, no obtuvo relación significativa con las relaciones interpersonales con la familia ( $\mathrm{r}=.072$ ) y entrenador $(\mathrm{r}=.127)$ y se relacionó positivamente con el resto de variables. La pasión armoniosa se relacionó positivamente con la inteligencia percibida en el juego $\left(\mathrm{r}=.524^{* *}\right)$ y el Índice de Autodeterminación $\left(\mathrm{r}=.391^{* *}\right)$. Asimismo, también lo hizo positivamente con la percepción de relaciones con la familia $\left(\mathrm{r}=.216^{* *}\right)$ y entrenador $(\mathrm{r}=$ .268**), y con la intención de práctica deportiva ( $\mathrm{r}=.438 * *)$, mientras que se relacionó inversamente con la percepción de conflicto entre el deporte y las otras actividades ( $\mathrm{r}=$ .315**). La pasión obsesiva tan solo mostró relaciones positivas con la inteligencia en el juego $\left(\mathrm{r}=.197^{*}\right)$ y la intención de práctica deportiva $(\mathrm{r}=292 * *)$.

Asimismo, el Índice de Autodeterminación estuvo relacionado positivamente con la inteligencia en el juego ( $\mathrm{r}=$ .391**), con la intención de práctica deportiva $\left(\mathrm{r}=.375^{* *}\right)$, y con la relación con el entrenador $\left(\mathrm{r}=.268^{* * *}\right)$ y la familia $(\mathrm{r}=$ .216**). Negativamente con la percepción de conflicto $(\mathrm{r}=$ .438**). La percepción de conflicto se relacionó negativamente con la intención de práctica deportiva $\left(r=-.485^{* *}\right)$ y con las relaciones con la familia $\left(\mathrm{r}=-.111^{*}\right)$.

\section{Análisis de mediación}

El análisis de mediación se realizó a través de la macro/ interfaz Process para SPSS. Se trabajó con 1000 muestras de bootstrapping y se incluyó después el test de Sobel. En primer lugar, se describen los resultados para el modelo de mediación con la pasión armoniosa. Se analizaron los efectos directos e indirectos de la motivación autodeterminada (variable independiente $\mathrm{X}$ ) y la pasión armoniosa (variable mediadora M) sobre la intención de práctica (variable dependiente Y). Los resultados del bootstrapping mostraron que el efecto de mediación descrito ha sido estadísticamente significativo (Boot LLCI = .1105 y Boot ULCI = .2480), comprobando así que el intervalo de confianza asociado no contiene el cero (Preacher \& Kelley, 2011). A su vez, los resultados del test de Sobel reflejan también que el modelo es estadísticamente significativo $(\mathrm{z}=4,16, \mathrm{p}<.001)$. También, se comprobaron los efectos directos e indirectos de la inteligencia en el juego (variable independiente X) y la pasión armoniosa (variable mediadora M) sobre la intención de prác- tica (variable dependiente Y). Los resultados del bootstrapping mostraron que el efecto de mediación descrito ha sido estadísticamente significativo (Boot LLCI = .1237 y Boot ULCI = .2962), comprobando así que el intervalo de confianza asociado no contiene el cero. A su vez, los resultados del test de Sobel reflejan también que el modelo es estadísticamente significativo $(\mathrm{z}=4,47, \mathrm{p}<.001)$. En segundo lugar, se describen los resultados para el modelo de mediación con la pasión obsesiva. Se analizaron los efectos directos e indirectos de la motivación autodeterminada (variable independiente $\mathrm{X}$ ) y la pasión obsesiva (variable mediadora M) sobre la intención de práctica (variable dependiente Y). Los resultados del bootstrapping mostraron que el efecto de mediación descrito ha no sido estadísticamente significativo (Boot LLCI = .0093 y Boot ULCI = .0670), comprobando así que el intervalo de confianza asociado si contiene el cero. A su vez, los resultados del test de Sobel reflejan también que el modelo no es estadísticamente significativo $(z=1,19, p=.2328)$. Además, se comprobaron los efectos directos e indirectos de la inteligencia en el juego (variable independiente X) y la pasión obsesiva (variable mediadora M) sobre la intención de práctica (variable dependiente Y). Los resultados del bootstrapping mostraron que el efecto de mediación descrito ha sido estadísticamente significativo (Boot LLCI = .0098 y Boot ULCI = .0865), comprobando así que el intervalo de confianza asociado no contiene el cero. A su vez, los resultados del test de Sobel reflejan también que el modelo es estadísticamente significativo ( $\mathrm{z}=$ 2,02, $\mathrm{p}=.0436$ ).

\section{Análisis estructural}

Se analizaron dos modelos con el método de Máxima verosimilitud y bootstrapping (1000 muestras). En ellos se plantearon las relaciones entre las variables inteligencia en el juego, motivación autodeterminada, pasión armoniosa u obsesiva, percepción de conflicto, relaciones con la familia y el entrenador e intención de práctica deportiva que se indican en las (figuras 1 y 2 ).

\section{Análisis del modelo 1 de la Pasión Armoniosa}

Se procedió a realizar el análisis de la normalidad multivariada. Se obtuvo una Kurtosis multivariante $(\mathrm{k}=1.375)$ y un coeficiente de critical ratio (c.r. = .819), inferior a 1,96, por lo que se aceptó el supuesto de normalidad.

Los resultados manifestaron que la Inteligencia en el juego predijo positivamente la pasión armoniosa $(\beta=, 39 ; p<$ .001) y la motivación autodeterminada $(\beta=.39 ; p<.001)$. La pasión armoniosa predijo positivamente las relaciones con la familia $(\beta=.20 ; p<.05)$ y con el entrenador $(\beta=.27 ; p<$ .001), también predijo una mayor intención de práctica $(\beta=$

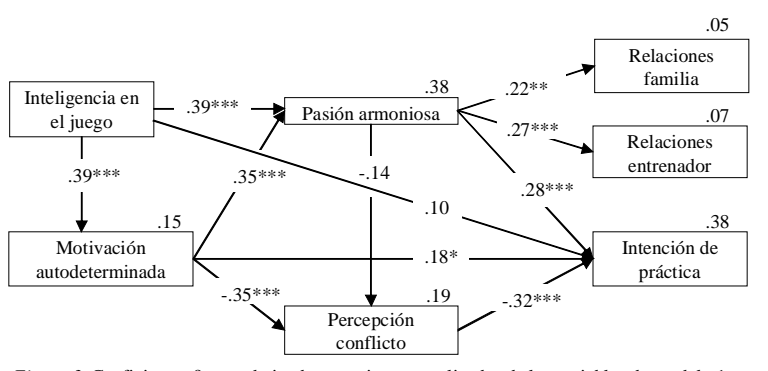

Figura 3. Coeficientes ß estandarizados y varianzas explicadas de las variables de modelo 1, con pasión armoniosa $(* * * p<.001 ; * * p<.01 ; * p<.05)$ 
$.28 ; p<.001)$, y estuvo muy cerca de predecir la percepción de conflicto relacionándose de forma negativa $(\beta=-.14 ; p=$ .069). La motivación autodeterminada predijo positivamente la pasión armoniosa $(\beta=.35 ; p<.001)$ y la intención de práctica $(\beta=.18 ; p<.05)$ y negativamente la percepción de conflicto $(\beta=-.35 ; p<.001)$. La percepción de conflicto predijo negativamente la intención de práctica deportiva $(\beta=$ .32; $p<.001)$.

Los índices de ajuste encontrados para el modelo fueron adecuados, ya que los índices de ajuste incrementales fueron mayores que $.90 \mathrm{y}$ el RMSEA obtuvo un valor por debajo de .08 (Hu \& Bentler, 1995). Los resultados fueron: $\chi^{2} / g l=$ $(19.018, \mathrm{gl}=10)=1.9 ; p=.040$; Incremental Fit Index $(\mathrm{IFI})=$ .966, Normative Fit Index (NFI) = .932, Comparative Fit Index $(\mathrm{CFI})=.965$ y Root Mean Square Error of Approximation $($ RMSEA $)=.071$.

\section{Análisis del modelo causal 2 de la Pasión Obsesiva}

Se procedió a realizar el análisis de la normalidad multivariada. Se obtuvo una Kurtosis multivariante $(\mathrm{k}=2.333)$ y un coeficiente de critical ratio (c.r. = 1.390), inferior a 1,96, por lo que se aceptó el supuesto de normalidad.

Los resultados del análisis mostraron, al igual que en el modelo anterior que la inteligencia en el juego predijo tanto la pasión obsesiva $(\beta=19 ; p<.05)$ como la motivación autodeterminada $(\beta=39 ; p<.001)$. Sin embargo, la motivación autodeterminada no predijo la pasión obsesiva $(\beta=02$; $p>$.05), aunque siguió prediciendo de forma positiva la intención de práctica y de forma negativa la percepción de conflicto. La pasión obsesiva no predijo ningún tipo de relaciones interpersonales, aunque sí predijo positivamente la intención de práctica $(\beta=26 ; p<.001)$. Finalmente, la percepción de conflicto predijo negativamente la intención de práctica ( $\beta=-.38 ; p<.001)$, al igual que en el modelo con pasión armoniosa. Los resultados mostraron que la pasión obsesiva no medió los efectos de la motivación autodeterminada sobre la intención de práctica deportiva.

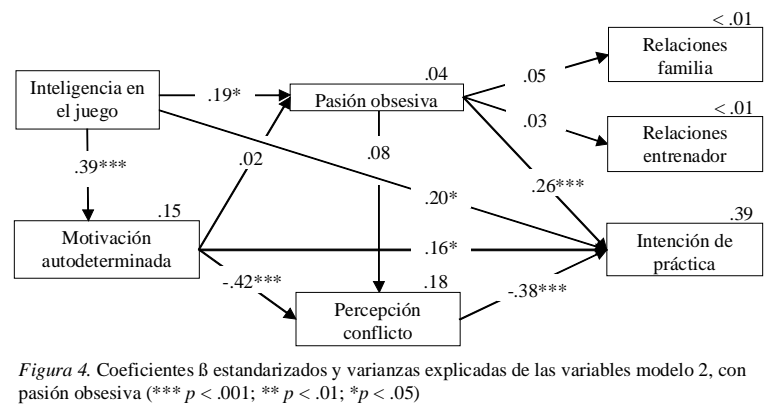

Los índices de ajuste para el modelo fueron aceptables, sin embargo mostraron menor ajuste que en el caso de la pasión armoniosa. Los resultados fueron: $\chi^{2} / g l=(27.429$; gl $=10)=2.74 ; p=.002$, Incremental Fit Index $($ IFI $)=.904$, Normative Fit Index $(\mathrm{NFI})=.856$, Comparative Fit Index $(\mathrm{CFI})$ $=.898$ y Root Mean Square Error of Approximation (RMSEA) $=.099$.

\section{Discusión}

En relación al primer objetivo de nuestro estudio, se confirma la hipótesis de que la pasión armoniosa actúa como mediadora entre la motivación autodeterminada y la intención de práctica y también, entre la inteligencia en el juego y la intención de práctica. En cambio, la pasión obsesiva medió entre la inteligencia en el juego y la intención de práctica pero no entre la motivación y la intención de práctica. En relación al segundo objetivo del estudio, en el que planteamos que el modelo de pasión armoniosa se relacionaría mejor con el resto de las variables y tendría un mejor ajuste que el modelo de pasión obsesiva., podemos decir que esta hipótesis también se confirmó. Los resultados indicaron que el modelo con pasión armoniosa presentó mejor ajuste que el modelo con pasión obsesiva puesto que ésta se relacionó con la motivación autodeterminada y con las relaciones interpersonales. Por todo ello, es razonable pensar que los deportistas con una pasión armoniosa alta se sienten más cómodos realizando la práctica deportiva porque están motivados de forma más autodeterminada, tienen menos conflictos con otras actividades (correlación negativa) y mantienen buenas relaciones interpersonales.

En relación al tercer objetivo de nuestro estudio, en el que planteamos que la inteligencia en el juego predeciría positivamente la pasión (armoniosa y obsesiva) y la motivación autodeterminada. La motivación autodeterminada predeciría positivamente sólo la pasión armoniosa, mientras que no predeciría la pasión obsesiva o lo haría negativamente, también predeciría positivamente la intención de práctica y negativamente la percepción de conflicto. La pasión armoniosa predeciría positivamente las relaciones interpersonales y la intención de práctica y negativamente la percepción de conflicto, mientras que la pasión obsesiva no predeciría las relaciones interpersonales o lo haría negativamente, sí predeciría la intención de práctica y predeciría positivamente la percepción de conflicto. Por último, la percepción de conflicto predeciría negativamente la intención de práctica. Podemos decir que esta hipótesis se confirma también casi en su plenitud. El estudio mostró que la inteligencia en el juego se relacionó de forma positiva con la motivación autodeterminada. Esta relación es relevante por sus implicaciones prácticas y puede ser debida a que las personas más inteligentes en el juego desarrollaron una mayor percepción de competencia y disfrutaron más de su práctica debido a una mejor comprensión de las situaciones que en ella se producen. El disfrute en la práctica permite a la persona participar plenamente en la actividad con una libertad propicia para generar experiencias positivas (Hodgins \& Knee, 2002).

Los resultados también mostraron que la inteligencia en el juego, se relacionó positivamente con los dos tipos de pasión. Al considerar las pasiones como actividades que han sido interiorizadas en la identidad de la persona (Deci \& Ryan, 1985; Koestner \& Losier, 2002), los deportistas son más inteligentes, ya que están muy familiarizados con la actividad. Es por ello que resuelven de una forma más fácil, efectiva e inteligente el contexto en el que se tiene que desenvolver. A su vez, mostró una relación más fuerte con la pasión armoniosa que con la pasión obsesiva. Cabe analizar en este punto el papel mediador de la motivación autodeterminada entre la inteligencia en el juego y la pasión armoniosa, que mostró que la inteligencia en el juego afectó a la pasión armoniosa no sólo de forma directa sino también 
de forma indirecta a través de la motivación autodeterminada, lo cual no ocurrió con la pasión obsesiva. Estos resultados concuerdan con el concepto de pasión armoniosa, ya que según Vallerand et al. (2003), ésta resulta de una autónoma internalización de la actividad en la identidad de la persona, que se produce cuando las personas tienen total libertad de acudir a la actividad, sin ningún tipo de presión.

La motivación autodeterminada también predijo negativamente la percepción de conflicto, de forma similar a los resultados obtenidos en estudios previos (Boiché \& Sarrazin, 2009; Guzmán \& Kingston, 2012). Los cuales han demostrado que el hecho de poseer una motivación autodeterminada hacia una actividad, hace que las personas sean menos susceptibles de percibir conflicto entre dicha actividad y otras actividades u otros roles sociales. En cambio, cuando la motivación es menos autodeterminada, el sujeto es más susceptible de experimentar este conflicto (Boiché \& Sarrazin, 2009), ya que se ejercitan por cualquier demanda externa o por conseguir algún tipo de recompensa (Deci \& Ryan, 2000). En esta línea, se han desarrollado varios trabajos que tratan de mostrar la existencia de un vínculo negativo entre la motivación autodeterminada y el conflicto de roles sociales por ejemplo (Ratelle, Vallerand, Senécal \& Provencher, 2005; Senécal, Julien \& Guay, 2003; Senécal, Vallerand \& Guay, 2001). Por lo tanto, una intención de práctica adecuada se caracterizaría por estar basada en una alta motivación autodeterminada y pasión armoniosa y una baja percepción de conflicto. En este sentido, la TAD aporta una base conceptual para explicar esta relación, estableciendo que la motivación autodeterminada actúa como un predictor del compromiso y la permanencia (Ntoumanis, 2005), mientras que la amotivación es causa del abandono (Sarrazin et al., 2002).

Los resultados de nuestros análisis mostraron que los dos tipos de pasión se relacionaron de forma positiva con la intención de práctica. Resultados similares obtuvieron Navarrón, Godoy-Izquierdo, Vélez, Ramírez-Molina \& Jiménez-Torres (2017) que indicaron que los jugadores con mayor pasión por el fútbol demostraron tener una mayor intención de práctica futura, de competición futura y de permanencia en el club. Pero se quiere aclarar que podrían actuar de forma muy diferente. Tanto si estás cómodo y eres libre realizando la actividad como si estás obsesionado con ella, vas a practicar mucho. Sin embargo, esta intención de práctica se acompañará de vivencias y emociones diferentes en función del tipo de pasión. En nuestro estudio, la percepción de las relaciones por parte de los jugadores manifiesta una disfunción o buena función en la relación con el entorno social. Diversas investigaciones muestran que los dos tipos de pasión pueden influir en las relaciones interpersonales, tanto dentro como fuera del contexto de la actividad deportiva (Lafreniere, Jowett, Vallerand \& Carbonneau, 2011). Sin embargo, los resultados del estudio mostraron que las relaciones con la familia y el entrenador se relacionaron positivamente con la pasión armoniosa. Esto está en concordancia con el modelo dualista de la Pasión (Vallerand et al., 2003) que postula que la pasión armoniosa conduce a tener menos conflictos interpersonales, mientras que la pasión obsesiva facilita el surgimiento de conflictos interpersonales. Sin embargo, en esta investigación la pasión obsesiva no guardó relación con las relaciones interpersonales, ni positiva ni negativa. También es cierto que la pasión obsesiva de los deportistas analizados no fue elevada, por lo que sería conveniente analizar el modelo en deportistas con una alta pasión obsesiva para ver si en este caso se verificaba la relación, este aspecto puede significar una limitación de la investigación. Por ello, podría hipotetizarse en futuros estudios, que la pasión obsesiva se acompaña de una mayor intención de práctica deportiva, a la vez que se percibe mayor conflicto con las relaciones sociales, el cual puede traducirse en mayor estrés y a la larga en el abandono de alguna de las actividades, ya sea deportivas o no. Además, de seguir indagando en si la pasión obsesiva influye positivamente en la percepción de conflicto, ya que no guardó ninguna relación en nuestra investigación. La que sí guardó una relación negativa y estuvo muy cerca de la significación fue la pasión armoniosa ( $\mathrm{p}=.069$ ), observando que puede existir una tendencia a que cuanto mayor sea la pasión armoniosa del deportista menor sería la percepción de conflicto con la actividad deportiva.

Por otro lado, existen algunas limitaciones en este estudio que hacen referencia a la imposibilidad de establecer relaciones causales por tratarse de un estudio correlacional, aunque si bien, por medio del modelo explicativo presentado, se favorece la comprensión de la relación existente entre diferentes variables que pueden influir en la intención de práctica y las relaciones interpersonales. Además, también es conveniente señalar que la medida de inteligencia en el juego se recoge mediante la percepción del jugador sobre su inteligencia y no sobre su inteligencia real.

Investigaciones futuras podrían estudiar el modelo en deportistas con alta pasión obsesiva para examinar específicamente las relaciones de este tipo de pasión cuando es alta y puede interferir en el funcionamiento cognitivoemocional-conductual adecuado. Además, se considera conveniente añadir en nuestro esquema estructural el flow disposicional como predictor de la intención futura de realizar actividad físico-deportiva (Franco et al., 2017). También podrían incluirse en este modelo el rendimiento deportivo, además de incorporar otras variables psicológicas como el estrés y la ansiedad, que podrían relacionarse en mayor medida con la pasión obsesiva y que podrían contribuir a predecir la aparición de lesiones por una práctica inadecuada o excesiva del deporte.

\section{Aplicaciones Prácticas}

Se piensa que, dados los resultados obtenidos, una buena forma de verificarlos y ponerlos en práctica sería realizar una intervención para mejorar la inteligencia en el juego, desarrollando deportistas más autodeterminados y apasionados, teniendo en cuenta el rendimiento del jugador (de forma objetiva).

A modo de conclusión, es importante desarrollar una adecuada inteligencia en el juego para un buen funcionamiento psicosocial del deportista, siendo la motivación autodeterminada y la pasión armoniosa dos variables mediadoras que aseguran un buen ajuste del deportista al entorno social y deportivo. Por ello, es apropiado emplear estrategias de intervención para mejorar la inteligencia en el juego como la utilización de técnicas de cuestionamiento (Gil \& Del 
Villar, 2014) o programas de supervisión reflexiva (Del Villar \& Iglesias, 2004) que ayuden a que el jugador optimice su capacidad de toma de decisiones y pueda incrementar su rendimiento deportivo (Del Villar \& Iglesias, 2004), así como recomendar a los entrenadores a orientar los entrenamientos buscando una mejora en la capacidad decisional del deportista en las diferentes acciones de juego (Conejero, Claver, Fernández-Echeverría, Gil-Arias, \& Moreno, 2017).Además, desarrollar la motivación autodeterminada a través de un clima de maestría y de autonomía (Ames, 1992), que fomenten la pasión armoniosa por el deporte. Así, los resultados de este estudio aportan bases para guiar intervenciones dirigidas a aumentar el disfrute y la adherencia a la práctica deportiva, lo que puede generar una mejora de la salud psicosocial y el rendimiento deportivo.

\section{Agradecimientos}

Me gustaría dar las gracias a todos los miembros del departamento de Educación física y deportiva y a la Universidad de Valencia por la financiación de esta investigación gracias a su programa de becas de iniciación a la investigación.

\section{Referencias}

Abraldes, J.A., Granero-Gallegos, A., Baena-Extremera, A., Gómez-López, M., y ～Rodríguez-Suárez, N. (2016). Orientaciones de meta, satisfacción, creencias de éxito y clima motivacional en nadadores. Revista Internacional de Medicina y Ciencias de la Actividad Física y el Deporte, 16(62), 583-599.

Almagro, B. J., \& Paramio-Pérez, G. (2017). Motivación y adherencia a la práctica de baloncesto en adolescentes. Cuadernos de Psicología del Deporte, 1(3), 189-198.

Almagro, B. J., Sáenz-López, P. \& Moreno, J. A. (2010). Prediction of sport adherence through the influence of autonomy-supportive coaching among Spanish adolescent athletes. Journal of Sports Science and Medicine, 9(1), 8-14.

Alonso, J. D., Rodríguez, V. D., Pérez, M. E. L., \& González, M.D.M.R. (2016). Motivación e inteligencia emocional en estudiantes de Educación Secundaria Obligatoria. Revista de Estudios e Investigación en Psicología y Educación, 3(2),94-101.

AMES, C. (1992): «Achievement goals, motiva-tional climate and motivational processes», enROBERTS, G.C.: Motivation in sport \& exercise. Champaign (Illinois). Human Kinetics,pp. 161-176

Baker, J., Côté, J. \& Abernethy, B. (2003). Sport specific training, deliberate practice and the development of expertise in team ball sports. Journal of Applied Sport Psychology, 15 (1), 12-25.

Bandura, A. (1997). Self-efficacy: the exercise of control. New York: W. H. Freeman and Company. Basic Books.

Bermejo, J. P., Almagro, B. J., \& Rebollo, J.A. (2018). Factores motivacionales relacionados con la intención de seguir practicando ejercicio físico en adultos y mayores. Retos - Nuevas Tendencias en Educación Física Deporte y Recreación, 31,(34), 117-122.
Boiché, J. C. \& Sarrazin, P. G. (2009). Proximal and distal factors associated with dropout versus maintained participation in organized sport. Journal of sports science \& medicine, 8(1), 9.

Brown, C. H., Gould, D. \& Foster, S. (2005). A framework for developing contextual intelligence (CI). The Sport Psychologist, 19(1), 51-62. doi:10.1123/tsp.19.1.51

Conejero, M., Claver, F., Fernández-Echeverría, C., Gil-Arias, A., \& Moreno, M. P. (2017). Toma de decisiones y rendimiento en las acciones de juego intermedias y finalistas en voleibol, en sets con diferente resultado. Retos - Nuevas Tendencias en Educación Física Deporte y Recreación, 31, 28-33.

Cortina, J. M. (1993). What is coefficient alpha? An examination of theory and applications. Journal of Applied Psychology, 78, 98-104. doi:10.1037/00219010.78.1.98

De la Vega, R., Del Valle, S., Maldonado, A. \& Moreno, A. (2008b). Pensamiento y Acción en el Deporte. Sevilla: Wanceulen.

Deci, E. L. \& Ryan, R. M. (2000). The» what» and» why» of goal pursuits: Human needs and the self-determination of behavior. Psychological inquiry, 11(4), 227-268. doi : 10.1207/s15327965pli1104_01

Deci, E. L., \& Ryan, R. M. (1985). Intrinsic motivation and self-determination in human behavior. New York: Plenum. http://dx.doi.org/10.1007/978-1-4899-2271-7

Del Villar, F. \& Iglesias, D. (2004). Conocimiento y toma de decisiones en jóvenes deportistas. Conferencia III Congreso Vasco del Deporte. Vitoria.

Elferink-Gemser, M.T., Visscher, C., Richard, H. \& Lemmink, K.A. P.M. (2005). Development of the tactical skills inventory for sports. Perceptual and Motor Skills, 99, 3, 883-895. doi :10.2466/pms.99.7.883-895

Ericsson, K. A. (2008). Deliberate practice and acquisition of expert perfomance: a general overview. Academic Emergency Medicine, 15, 988-994. doi:10.1111/j.15532712.2008.00227.x

Escartí, A. (2003). Socialización deportiva. En A. Hernández Mendo (Ed.). Psicología del Deporte (Vol. 1, pp. 88-104). Buenos Aires: Efdeportes.

Fernández Muñoz, J., \& García González, J. (2017). El análisis de mediación a través de la macro/interfaz Process para SPSS. REIRE Revista d'Innovació i Recerca en Educació, 10(2), 79-88.

Fernández-Ozcorta, E.,Almagro, B., \& Sáenz-López, P. (2017). Reseña de libro: Motivación, inteligencia emocional y actividad física en universitarios. Revista De Educación, Motricidad e Investigación, 0(6), 79-80.

Fontes, S., García-Gallego, C., Quintanilla, L., Rodríguez, R., Rubio de Lemus, P. \& Sarriá, E. (2010). Fundamentos de investigación en Psicología. Madrid: U n i versidad Nacional de Educación a Distancia.

Franco, E., Coterón, J., Gómez, V., Brito, J., \& Martínez, H. A. (2017). Influenciadela motivación y del flow disposicional sobre la intención de realizar actividad físico-deportiva en adolescentes de cuatro países. Retos. Nuevas Tendencias en Educación Física Deporte y Recreación, 31, 46-51

García, T., Leo, F. M., Martín, E. \& Sánchez, P. A. (2008). El compromiso deportivo y su relación con factores 
disposicionales y situacionales de la motivación. RICYDE. Revista Internacional de Ciencias del Deporte, 12(4), 45-58. doi:10.5232/ricyde2008.01203

García, V. (2009). Inteligencia contextual, competencia decisional, inteligencia emocional y habilidades de afrontamiento en deportistas de diferente nivel de pericia. Tesis doctoral inédita, Universidad de Castilla - La Mancha, Toledo.

Gardner, H. (1983). Frames of mind: The theory of multiple intelligences. New York: Basic books. doi : 10.1177/ 001698628502900212

Gardner, H. (1983). Frames of mind: The theory of Multiple Intelligences. New Cork:

Gardner, H. (2008). Inteligencias múltiples. La teoría en la práctica. Barcelona: Paidós.

Gardner, H. (2010). La inteligencia reformulada: las inteligencias múltiples en el siglo XXI. Barcelona: Paidós.

Gil, A., \& del Villar, F. (2014). Aplicación de un programa de entrenamiento decisional, en tiempo real de juego, para la mejora de rendimiento táctico individual del deportista. En L. García-González y F. Del-Villar (Eds.), Entrenamiento táctico y decisional en el deporte (pp. 132-145). Madrid: Síntesis.

Gutiérrez, M., Tomás, J. M., \& Calatayud, P. (2018). Determinantes de la práctica deportiva de los adolescentes en horario extraescolar. Revista Iberoamericana de Psicología del Ejercicio y el Deporte, 13(1), 91-100. doi: 10.17979/sportis.2017.3.1.1747

Guzmán, J. F. \& Kingston, K. (2011). Prospective study of sport dropout: A motivational analysis as a function of age and gender. European Journal of Sport Science, 12 (5), 431-442. doi:10.1080/17461391.2011.573002

Guzmán, J. F., Carratalá, E., García-Ferriol, A., \& Carratalá, V. (2010). Propiedades psicométricas de una escala de motivación deportiva. Motricidad. European Journal of Human Movement, 16, 85-98.

Hatfield, B. D., \& Kerick, S. E. (2007). The psychology of superior sport performance: A cognitive and affective neuroscience perspective. En G. Tenenbaum y R.C. Eklund (Eds.), Handbook of Sport Psychology (3rd Edition) (pp 84-112). New Jersey: John Wilet \& sons. http://dx.doi.org/10.1002/9781118270011.ch4

Hayes, A. F. 2012. PROCESS: A versatile computational tool for observed variable $\quad m e d i a t i o n$, moderation, and conditional process modeling [White Paper]. Retrieved from http://www.afhayes.com/ index.html

Hodgins, H. S. \& Knee, C. R. (2002). The integrating self and conscious experience. In E. L. Deci y R. M. Ryan (Eds.), Handbook of self-determination research (pp. 87-100). Rochester, NY: University Of Rochester Press.

Hu, L. y Bentler, P. M. (1995). Evaluating model fit. En R. Hoyle (Ed.), Structural equation modeling: Issues, concepts, and applications (pp. 76- 99). Newbury Park, CA: Sage.

Jones, G. (2012). The role of Superior Performence Intelligence in sustained success. En S.M. Murphy (Ed.), The Oxford Handbook of Sport and Performance Psychology (pp. 62-80). Oxford: Oxford University Press. http:// dx.doi.org/10.1093/oxfordhb/9780199731763.013.0004

Koestner, R., Losier, GF., Vallerand, R.J., \& Carducci, D. (1996).
Identified and introjected forms of political internalization: Extending self-determination theory. Journal of Personality and Social Psychology, 70, 1025-1036.

Lafrenie're, M. A. K., Jowett, S., Vallerand, R. J., Donahue, E. G., \& Lorimer, R. (2008). Passion in sport: On the quality of the coach-athlete relationship. Journal of Sport \& Exercise Psychology, 30, 1-22.

Lafreniere, M., Jowett, S., Vallerand, R. \& Carbonneau, N. (2011). Passion for coaching and the quality of the coach - athlete relationship :The mediating role of coaching behaviors. Psychology of Sport \& Exercise, 12 (2), 144152. doi:10.1016/j.psychsport.2010.08.002

Luckwü, R. M. \& Guzmán, J. F. (2011). Deportividad en balonmano: un análisis desde la Teoría de la Autodeterminación. Revista de Psicología del Deporte, 20 (2), 305-320.

Martín, M., Guzmán, J. F. \& De Benito, A.M. (2018). Inteligencia emocional, percepción de apoyo a la autonomía y relaciones en el deporte. Cuadernos de Psicología del Deporte, 18(1), 13-20.

Medrano, E. F., \& Mateos, M. E. (2018). Evaluación de la motivación en adolescentes que practican deporte en edad escolar (Evaluation of motivation in teenagers who practice sports in school age). Retos - Nuevas Tendencias en Educación Física Deportey Recreación, (33), 27-33.

Méndez-Giménez, A., Cecchini, J. A., \& Fernández-Río, J. (2016). Pasión por el deporte, actividad física vigorosa y satisfacción con la vida. Revista de Psicología Del Deporte, 25(1)

Muñoz, S. P., Calle, R. C., Muñoz, A. S., Cayetano, A. R., de Mena, J. M., Ramos, J. M. F., \& Sáez, C. C. (2018). ¿Por qué juego al fútbol si soy una mujer?: Motivaciones para jugar al fútbol. Retos - Nuevas. Tendencias en Educación Física Deporte y Recreación, 34, 183-188.

Navarrón, E., Godoy-Izquierdo, D., Vélez, M., Ramírez-Molina, M. J., \& Jiménez-Torres, M. G (2017). Implementación de una intervención psicológica en fútbol base, satisfacción subjetiva de los deportistas y experiencias de pasión, competencia percibida y compromiso deportivo en relación con la intención de práctica futura. Revista iberoamericana de psicología del ejercicio y el deporte, 12(1), 59-69.

Ntoumanis, N. (2005). Aprospective study of participation in optional school physical education using a selfdetermination theory framework. Journal of Educational Psychology, 97 (3), 444 - 453. doi:10.1037/00220663.97.3.444

Olympiou, A., Jowett, S. \& Duda, J. L. (2008). The psychological interface between the coach-created motivational climate and the coach-athlete relationship in team sports. The Sport Psychologist, 22, 423-438. doi:10.1123/tsp.22.4.423

Ommundsen, Y., \& Vaglum, P. (1997). Competence, perceived competence and drop-out from soccer: a study of young playeres. Scandinavian Journal of Medecine \& Science in Sports, 7, 6, 373-383. http://dx.doi.org/10.1111/ j.1600- 0838.1997.tb00170.x

Pedrosa, I., García-Cueto, E., Torrado, J. \& Arce, C. (2017). Adaptación Española de la Escala de Pasión al Ámbito Deportivo. Revista Iberoamericana de Diagnóstico y 
Evaluación Psicológica, 1 (43), p. 165-176. doi : 10.21865/ ridep43_16

Pelletier, L, Fortier, M, Vallerand, R. \& Brière, N. (2001). Associations among perceived autonomy support, forms of self-regulation, and persistence: A prospective study. Motivation and Emotion, 25, 279-306.

Pelletier, L. G., Rocchi, M. A., Vallerand, R. J., Deci, E. L. \& Ryan, R. M. (2013). Validation of the revised sport motivation scale (SMS-II). Psychology of Sport and Exercise, 14, 329-341. doi:10.1016/ j.psychsport.2012.12.002

Pérez, L. M., Navia, J., Espín, J. M., Coll, M. V., \& Nieto, M. P. (2015). Autopercepción de inteligencia contextual para jugar y de competencia decisional en el fútbol. RICYDE. Revista Internacional de Ciencias del Deporte, 11(42), 329-338.

Philippe, F. L., Vallerand, R. J., Houlfort, N., Lavigne, G. \& Donahue, E. G. (2010). Passion for an activity and quality of interpersonal relationships: The mediating role of emotions. Journal of Personality and Social Psychology, 98 (6), 917-932. doi:10.1093/acprof:oso/ 9780199777600.003.0011

Philippe, F. L., Vallerand, R. J., Houlfort, N., Lavigne, G., \& Donahue, E. G. (2010). Passion for an activity and quality of interpersonal relationships: The mediating role of emotions. Journal of Personality and Social Psychology, 98, 917-932.

Philippe, F. L., Vallerand, R. J., Houlfort, N., Lavigne, G., \& Donahue, E. G. (2010). Passion for an activity and quality of interpersonal relationships: The mediating role of emotions. Journal of Personality and Social Psychology, 98, 917-932.

Preacher, K. J., \& Kelley, K. (2011). Effect Size Measures for Mediation Models: Quantitative Strategies for Communicating Indirect Effects. Psychological Methods, 16(2), 93 - 115

Ratelle, C. F., Vallerand, R. J., Senécal, C., \& Provencher, P. (2005). The relationship between school-leisure conflict and educational and mental health indexes: A motivational analysis. Journal of Applied Social Psychology, 35(9), 1800-1823.

Rodríguez Allen, A. (2000). Adolescencia y deporte. Oviedo: Nobel.

Ruiz, L. M, Navia, J., Miñano, J., García, V. \& Palomo, M. (2015). Autopercepción de inteligencia contextual para jugar y de competencia decisional en el fútbol. RICYDE. Revista Internacional de Ciencias del Deporte, 11 (42), 329-338. doi:10.5232/ricyde2015.04202

Ruiz, L. M., García, V., Palomo, M., Navia, J.A. \& Miñano, J. (2014). Inteligencia contextual y pericia en el fútbol. Revista Internacional de Medicina y Ciencias de la Actividad Física y el Deporte, 54, 307-317.

Ruiz, L. M., Graupera, J. L. \& García, V. (2014). Inteligencia contextual percibida en el deporte. Desarrollo y validación de un cuestionario. Cultura, Ciencia y Deporte: Revista de ciencias de la actividad física y del deporte, 27, 211-224.

Ruiz, L., Navia, J., Miñano, J., García, V. \& Palomo, M. (2015). Autopercepción de inteligencia contextual para jugar y de competencia decisional en el fútbol. RICYDE. Revista Internacional de Ciencias del Deporte, 11(42), 329-338.

Ruiz-Juan, F., \& Zarauz, A. (2012a). Predictor variables of motivation in Spanish master athletes. Journal of
Human Sport and Exercise, 7(3), 617-628.

Sarrazin, P., Vallerand, R., Guillet, E., Pelletier, L. \& Cury, F. (2002). Motivation and dropout in female handballers: A 21 month prospective study. European Journal of Social Psychology, 32 (3), 395-418.

Se'guin-Le'vesque, C., Laliberte', M. L., Pelletier, L. G., Vallerand, R. J., \& Blanchard, C. (2003). Harmonious and obsessive passions for the internet: Their associations with couples' relationships. Journal of Applied Social Psychology, 33, 197-221.

Senécal, C., Julien, E., \& Guay, F. (2003). Role conflict and academic procrastination:A selfdetermination perspective. European Journal of Social Psychology, 33, 135-145.

Senecal, C., Vallerand, R. J., \& Guay, F. (2001). Antecedents and ' outcomes of work- family conflict: Toward a motivational model. Pers. Soc. Psychol. Bull. 27: 176186.

Sternberg, R. J. (1985). Beyond IQ: A Triarchic Theory of Intelligence. Cambridge: Cambridge University Press.

Sternberg, R. J. (2000). Intelligence and wisdom. En R. Sternberg (Ed.), Handbook of Intelligence (pp. 631649). New York: Cambridge University Press. http:// dx.doi.org/10.1017/cbo9780511807947.029

Sternberg, R. J., Forsythe, G. B., Hedlund, J., Horvath, J. A., Wagner, R. K., Williams, W. M., Snook, S. A. \& Grigorenko, E. L. (2000). Practical intelligence in everyday life. New York: Cambridge University Press.

Terenzini, P. T. (1993). On the nature of Institutional Research and the knowledge and skills, it requires. Research in Higher Education, 34, 1, 1-10. http://dx.doi.org/ 10.1007/BF00991859

Vallerand, R. J. (2010). On passion for life activities: The Dualistic Model of Passion. Advances in Experimental Social Psychology, 42, 97-193.

Vallerand, R. J. (2012).The role of passion is sustainable psychological well-being.Psychology of Well-Being: Theory, Research and Practice, 2(1).

Vallerand, R. J., Ntoumanis, N., Philippe, F., Lavigne, G. L., Carbonneau, C., Bonneville, A., et al. (2008). On passion and sports fans: A look at football. Journal of Sport Sciences, 26, 1279-1293.

Vallerand, R.J. \& Houlfort, N. (2003). Passion at work: Toward a new conceptualization. En D. Skarlicki, S. Gilliland y D. Steiner (Eds.), Social issues in management (pp. 175204). (Vol. 3). Greenwich, CT: Information Age Press.

Vallerand, R.J. (2007). Intrinsic and Extrinsic Motivation in Sport and Physical Activity. A Review and a look at the future. En G. Tenenbaum y R. C. Eklund (Eds.), Handbook of sport Psychology (3a ed., pp. 59-83). New York: Wiley. doi:10.1002/9781118270011.ch3

Vallerand, R.J. (2008). On the psychology of passion: in search of what makes people's lives most worth living. Canadian Psychology, 49 (1), 1-13. doi:10.1037/0708-5591.49.1.1

Ward, P., Hodges, N. J., Williams, A. M. \& Starkes, J. L. (2004). Deliberate practice and expert performance: Defining the path to excellence. In A. M. Williams and N. J. Hodges (Eds.), Skill acquisition in sport: Research, theory and practice (pp. 231 - 258). London: Routledge.

Zubiaur, M. \& Riego, M. (2015). Motivos e intereses de practicantes de BMX adolescentes: Un estudio piloto. Retos Nuevas tendencias en Educación Fiìsica, Deporte y Recreacioìn, 27, 109-113. 